\title{
Reallocation Problems in Scheduling
}

\author{
Michael A. Bender \\ Computer Science, \\ Stony Brook University \\ and Tokutek, Inc. \\ USA \\ bender@cs.stonybrook.edu
}

\author{
Martin Farach-Colton \\ Computer Science, \\ Rutgers University \\ and Tokutek, Inc. \\ USA \\ farach@cs.rutgers.edu
}

\author{
Sándor P. Fekete \\ Computer Science \\ TU Braunschweig \\ Germany \\ s.fekete@tu-bs.de
}

\author{
Jeremy $\mathrm{T}$. Fineman \\ Computer Science \\ Georgetown University \\ USA \\ jfineman@cs.georgetown.edu
}

\author{
Seth Gilbert \\ Computer Science \\ National University of Singapore \\ Singapore \\ seth.gilbert@comp.nus.edu.sg
}

\begin{abstract}
In traditional on-line problems, such as scheduling, requests arrive over time, demanding available resources. As each request arrives, some resources may have to be irrevocably committed to servicing that request. In many situations, however, it may be possible or even necessary to reallocate previously allocated resources in order to satisfy a new request. This reallocation has a cost. This paper shows how to service the requests while minimizing the reallocation cost.

We focus on the classic problem of scheduling jobs on a multiprocessor system. Each unit-size job has a time window in which it can be executed. Jobs are dynamically added and removed from the system. We provide an algorithm that maintains a valid schedule, as long as a sufficiently feasible schedule exists. The algorithm reschedules only $O\left(\min \left\{\log ^{*} n, \log ^{*} \Delta\right\}\right)$ jobs for each job that is inserted or deleted from the system, where $n$ is the number of active jobs and $\Delta$ is the size of the largest window.
\end{abstract}

\section{Categories and Subject Descriptors}

F.2.2 [Analysis of Algorithms and Problem Complexity]: Nonnumerical Algorithms and Problems-Sequencing and scheduling

\section{Keywords}

Scheduling, online problems, reallocation

\footnotetext{
This research was supported in part by NSF grants IIS 1247726 , IIS 1247750, CCF 1114930, CCF 1217708, CCF 1114809, CCF 0937822, CCF 1218188, and by Singapore NUS FRC R-252-000443-133.
}

Permission to make digital or hard copies of all or part of this work for personal or classroom use is granted without fee provided that copies are not made or distributed for profit or commercial advantage and that copies bear this notice and the full citation on the first page. Copyrights for components of this work owned by others than ACM or the author must be honored. To copy otherwise, or republish, to post on servers or to redistribute to lists, requires prior specific permission and/or a fee.

SPAA'13, June 23-25, 2013, Montréal, Québec, Canada.

Copyright 2013 ACM 978-1-4503-1572-2/13/07 ...\$15.00.

\section{INTRODUCTION}

Imagine you are running a doctor's office. Every day, patients call and try to schedule an appointment, specifying a time period in which they are free. You respond by agreeing to a specific appointment time. Sometimes, however, there is no available slot during the period of time specified by the patient. What should you do? You might simply turn the patient away. Or, you can reschedule some of your existing patients, making room in the schedule. ${ }^{1} \mathrm{Un}$ fortunately, patients do not like being rescheduled. How do you minimize the number of patients whose appointments are rescheduled?

While scheduling a doctor's office may (or may not) seem a somewhat contrived motivating example, this situation arises with frequency in real-world applications. Almost any scenario that involves creating a schedule also requires the flexibility to later change that schedule, and those changes often have real costs (measured in equipment, computation, or tempers). For example, in the computational world, scheduling jobs on multiprocess machines and scheduling computation on the cloud lead to rescheduling. In the physical world, these problems arise with depressing regularity in scheduling airports and train stations. Real schedules are always changing.

In a tightly packed schedule, it can be difficult to perform this rescheduling efficiently. Each task you reschedule risks triggering a cascade of other reschedulings, leading to high costs (and unhappy patients). It is easy to construct an example where each job added or removed changes $\Omega(n)$ other jobs, even with constantsized tasks. In this paper, we show that if there is slack in the schedule, then these rescheduling cascades can be collapsed, in fact down to $O\left(\log ^{*} n\right)$ for unit-size jobs.

\section{Reallocation Problems}

We introduce a framework for studying the familiar topic of how to change resource allocations as problem instances change, with a goal of unifying results of this type, e.g., [16, 26, 29]. We call problems in this framework reallocation problems. A reallocation problem is online in the sense that requests arrive and the system responds. Unlike in the standard online setting where resources

\footnotetext{
${ }^{1}$ Before you get too skeptical about the motivation, this is exactly what M. F-C's ophthalmologist does.
} 
are irrevocably assigned, in a reallocation problem, allocations may change. These reallocations, however, have a cost.

Reallocation lies somewhere between traditional notions of offline and online resource allocation. If the reallocation cost is 0 , then there is no penalty for producing an optimal allocation after each request. In this case, a reallocation problem can be viewed as a sequence of offline problems. If the cost of reallocation is $\infty$, then no finite-cost reallocation is possible and the result is a traditional online problem. When there is a bounded but non-zero cost for reallocation, then there is a trade-off between the quality of an allocation and the cost of reallocation.

Many related questions have been asked in the scheduling community (explored more fully below), including: how can one design schedules that are robust to uncertain or noisy inputs (see, e.g., [22, 24]); how can one generate schedules that change in a limited way while still remaining close to optimal [28]; what is the computational cost of finding a new optimal schedule as the inputs change (e.g., $[1,2,4,10])$. Our approach differs in that it is jobcentered, meaning that we measure the cost of moving jobs rather than the cost of computing where jobs should move to.

Reallocation is a natural problem. Many existing algorithms, when looked in the right way, can be viewed as reallocation problems, e.g., reconfiguring FPGAs [14], maintaining a sparse array [9, 17,31-33], or maintaining an on-line topological ordering (e.g., [8, $15,21])$. We believe that the framework developed in this paper will allow us to achieve new insights into classical scheduling and optimization problems and the cost of changing a good solution when circumstances change.

\section{Our Problem}

We focus on the reallocation version of a classical multiprocessor scheduling problem [18] (described more fully in Section 2). We are given a set of unit-length jobs to process on $m$ machines. Each job has an arrival time and a deadline. The job must be assigned to a machine and processed at some point within the specified time window. Jobs are added and removed from the schedule dynamically. The goal is to maintain a feasible schedule at all times.

In order to process a request, it may be necessary to reschedule some previously scheduled jobs. There are two ways in which a job may be rescheduled: it may be reallocated to another time on the same machine, or it may be migrated to a different machine. The migration cost is the total number of jobs that are moved to different machines when new jobs are added or removed. The reallocation cost is the total number of jobs that are rescheduled, regardless of whether they are migrated or retained on the same machine. Our goal is to minimize both the migration cost and the total reallocation cost. We bound these costs separately, since we expect that a reallocation might be more expensive if it also entails a migration. (See [5,7] for other work that considers migrations separately from other scheduling considerations, such as preëmptions.)

We call an algorithm that processes such a sequence of scheduling requests a reallocating scheduler. We show in Section 6 that a reallocating scheduler must allow for some job migrations and that there is no efficient reallocating scheduler without some form of resource augmentation; here we consider speed augmentation [20, 25]. We say that an instance is $\boldsymbol{\gamma}$-underallocated if it is feasible even when all jobs sizes (processing times) are multiplied by $\gamma$. In other words, the offline scheduler is $\gamma$ times slower than the online scheduler.

\section{Results}

This paper gives an efficient $m$-machine reallocating scheduler for unit-sized jobs with arrival times and deadlines. Informally, the pa- per shows that as long as there is sufficient slack (independent of $m$ ) in the requested schedule, then every request is fulfilled, the reallocation cost is small, and at most one job migrates across machines on each request. Specifically, this paper establishes the following theorem:

THEOREM 1. There exists a constant $\gamma$ as well as a reallocating scheduler for unit-length jobs such that for any m-machine $\gamma$-underallocated sequence of scheduling requests, we achieve the following performance. Let $n_{i}$ denote the number of jobs in the schedule and $\Delta_{i}$ the largest window size when the ith reallocation takes place. Then the ith reallocation

- has cost $O\left(\min \left\{\log ^{*} n_{i}, \log ^{*} \Delta_{i}\right\}\right)$, and

- requires at most one machine migration.

We prove Theorem 1 in stages. In Sections 3 and 4, we assume that job windows are all nicely "aligned," by which we mean that all job windows are either disjoint, or else one is completely contained in the other. In Section 3, we show that the multi-machine aligned case can be reduced to the single-machine aligned case, sacrificing a constant-factor in the underallocation. In Section 4, we establish Theorem 1, assuming the windows are aligned and that $m=1$. Finally, in Section 5, we remove the alignment assumption from Section 4, again sacrificing a constant-factor in the underallocation.

The crux of our new approach to scheduling appears in Section 4. This section gives a simple scheduling policy that is robust to changes in the scheduling instances. By contrast, most classical scheduling algorithms are brittle, where small changes to a scheduling instance can lead to a cascade of job reallocations even when the system is highly underallocated. This brittleness is certainly inherent to earliest-deadline-first (EDF) and least-laxity-first (LLF) scheduling policies, the classical greedy algorithms for scheduling with arrival times and deadlines. In fact, we originally expected that any greedy approach would necessarily be fragile. We show that this is not the case.

Our new scheduler is based upon a simple greedy policy ("reservation-based pecking-order scheduling"). Unlike most robust algorithms, which explicitly engineer redundancy, the resiliency of our scheduler derives from a basic combinatorial property of the underlying "reservation" system. In this sense, it feels different from typical mechanisms for achieving robustness in computer science or operations research.

\section{Related Work}

Here, we flesh out the details of related scheduling and resource allocation work.

Robust scheduling (or "robust planning") involves designing schedules that can tolerate some level of uncertainty. See [22, 24] for surveys and $[11,12,19,23]$ for applications to train and airline scheduling. The assumption in these papers is that the problem is approximately static, but there is some error or uncertainty, or that the schedule remains near optimal even if the underlying situation changes [28]. By contrast, we focus on an arbitrary, worst-case, sequence of requests that may lead to significant changes in the overall allocation of resources.

Researchers have also focused on finding a good fall-back plan ("reoptimization") when a schedule is forced to change. Given an optimal solution for an input, the goal is to compute a near-optimal solution to a closely related input $[1,3,4,10]$. These papers typically focus on the computational complexity of incremental optimization. By contrast, we focus on the cost of changing the schedule.

Shachnai et al. [27] introduced a framework that is most closely related to ours. They considered computationally intractable prob- 
lems that admit approximation algorithms. When the problem instance changes, they would like to change the solution as little as possible in order to reestablish a desired approximation ratio. One difference between their framework and ours is that we measure the ratio of reallocation cost to allocation cost, whereas there is no notion of initial cost for them. Rather they measure the ratio of the transition cost to the optimal possible transition cost that will result in a good solution. Although their framework is an analogous framework for approximation algorithms, the particulars end up being quite different.

Davis et al. [13] propose a resource reallocation problem where the allocator must assign resources with respect to a userdetermined set of constraints. The constraints may change, but the allocator is only informed when the solution becomes infeasible. The goals is to minimize communication between the allocator and the users.

Many other papers in the literature work within similar setting of job reallocations, but with different goals, restrictions, or scheduling problems in mind. Unal et al. [29] study a problem wherein an initial feasible schedule consisting of jobs with deadlines must be augmented to include a set of newly added jobs, minimizing some objective function on only the new jobs without violating any deadline constraints on the initial schedule. As in the present paper they observe that slackness in the original schedule facilitates a more robust schedule, but outside of the hard constraints they do not count the reallocation cost. Hall and Potts [16] allow a sequence of updates and aim to restrict the change in the schedule, but they evaluate the quality of their algorithm incrementally rather than with respect to a full sequence of updates or an offline objective.

More closely related to our setting, Westbrook [30] considers the total cost of migrating jobs across machines in an online loadbalancing problem while also keeping the maximum machine load competitive with the current offline optimum, which is a different scheduling problem in a similar framework. Unlike in the present paper, Westbrook considers only migration costs and does not include the reallocation cost of reordering jobs on machines. Sanders et al. [26] consider a similar load-balancing problem with migration costs and no reallocation costs; their goal is to study the tradeoff between migration costs and the instantaneous competitive ratio.

\section{REALLOCATION MODEL}

Formally, an on-line execution consists of a sequence of scheduling requests of the following form: $\langle$ INSERTJOB, name, arrival, deadline $\rangle$ and 〈DELETEJoB, name $\rangle$. A job $j$ has integral arrival time $a_{j}$ and deadline $d_{j}>a_{j}$, meaning that it must be scheduled in a timeslot no earlier than time $a_{j}$ and no later than time $d_{j}$. We call the time interval $\left[a_{j}, d_{j}\right]$ the job's window $W$. We call $d_{j}-a_{j}$, denoted by $|W|$, the window $W$ 's span. We use job $\boldsymbol{j}$ 's span as a shorthand for its window's span. Each job takes exactly one unit of time to execute.

At each step, we say that the active jobs are those that have already been inserted, but have not yet been deleted. Before each scheduling request, the scheduler must output a feasible schedule for all the active jobs. A feasible schedule is one in which each job is properly scheduled on a particular machine for a time in the the job's available window, and no two jobs on the same machine are scheduled for the same time. Notice that we are not concerned with actually running the schedule; rather, we construct a sequence of schedules subject to an on-line sequence of requests.

We define the migration cost of a request $r_{i}$ to be the number of jobs whose machine changes when $r_{i}$ is processed. We define the reallocation cost of a request $r_{i}$ to be the number of jobs that must be rescheduled when $r_{i}$ is processed.
When the scheduling instances do not have enough "slack" it may become impossible to achieve low reallocation costs. In fact, if there are $n$ jobs currently scheduled, a new request may have reallocation cost $\Theta(n)$. Even worse, it may be that most reallocations require most jobs to be moved, as is shown in Lemma 12: for largeenough $s$, there exist length- $s$ request sequences, in which $\Theta\left(s^{2}\right)$ reallocations are necessary. Moreover, for large-enough $s$, there exist length- $s$ request sequences in which $\Theta(s)$ machine migrations are necessary (see Lemma 11).

\section{Underallocated Schedules and Our Result}

To cope with Lemmas 11 and 12, we consider schedules that contain sufficient slack, i.e., that are not fully subscribed. We say that a set of jobs is $\boldsymbol{m}$-machine $\boldsymbol{\gamma}$-underallocated, for $\gamma \geq 1$, if there is a feasible schedule for those jobs on $m$ machines even when the job length (processing time) is multiplied by $\gamma$. This is equivalent to giving the offline scheduler a processing speed that is $\gamma$ times slower than the online scheduler. When $m$ is implied by context, we simply say $\gamma$-underallocated.

Overloading terminology, we say that a sequence of scheduling requests is $\gamma$-underallocated if after each request the set of active jobs is $\gamma$-underallocated.

\section{Aligned-Windows Assumption}

The assumption of aligned windows is used in Sections 3 and 4, but it is dropped in Section 5 to prove the full theorem. We say that a window $W$ is aligned if (i) it has span $2^{i}$, for some integer $i$, and (ii) it has a starting time that is a multiple of $2^{i}$. If a job's window is aligned, we say that the job is aligned. We say that a set of windows (or jobs) are recursively aligned if every window (or job) is aligned.

Notice that recursive alignment implies that two jobs windows are either equal, disjoint, or one is contained in the other (i.e., the windows are laminar). Dealing with recursively aligned windows is convenient in part due to the following observation.

LEMMA 2. If a recursively aligned set of jobs is m-machine $\gamma$ underallocated, then for any aligned window $W$ there are at most $m|W| / \gamma$ jobs with span at most $|W|$ whose windows overlap $W$.

Proof. The window $W$ comprises $|W|$ timeslots on each of $m$ machines, for a total of $m|W|$ timeslots. By definition, a $\gamma$-underallocated instance is feasible even if the jobs' processing times are increased to $\gamma$. Thus, there may be at most $m|W| / \gamma$ jobs restricted to window $W$. Since the set of jobs is recursively aligned, if a job has window $W^{\prime}$ that overlaps $W$ and $\left|W^{\prime}\right| \leq|W|$, then $W^{\prime}$ is fully contained by $W$. Hence, there can be at most $m|W| / \gamma$ such jobs.

\section{REALLOCATING ALIGNED JOBS ON MULTIPLE MACHINES}

This section algorithmically reduces the multiple-machine scheduling problem to a single-machine scheduling problem, assuming recursive alignment. The reduction uses at most one migration per request. We use $m$ to denote the number of machines.

The algorithm is as follows. For every window $W$, record the number $n_{W}$ of jobs having window $W$. (This number need only be recorded for windows that exist in the current instance, so there can be at most $n$ relevant windows for $n$ jobs.) The goal is to maintain the invariant that every machine has between $\left\lfloor n_{W} / m\right\rfloor$ and $\left\lceil n_{W} / m\right\rceil$ jobs with window $W$, with the extra jobs being assigned to the earliest machines. This invariant can be maintained simply by delegating jobs, for each window $W$, round-robin: if 
there are $n_{W}$ jobs with window $W$, a new job with window $W$ is delegated to machine $\left(n_{W}+1\right) \bmod m$. When a job with window $W$ is deleted from some machine $m_{i}$, then a job is removed from machine $\left(n_{w} \bmod m\right)$ and migrated to machine $m_{i}$. All job movements are performed via delegation to the single-machine scheduler on the specified machine(s).

The remaining question is whether the instances assigned to each machine are feasible. The following lemma says that they are.

LEMMA 3. Consider any m-machine $6 \gamma$-underallocated recursively aligned set of jobs $J$, where $\gamma$ is an integer. Consider a subset of jobs $J^{\prime}$ such that if $J$ contains $n_{W}$ jobs of window $W$, then $J^{\prime}$ contains at most $\left\lceil n_{W} / m\right\rceil$ jobs of window $W$. Then $J^{\prime}$ is 1 machine $\gamma$-underallocated.

Proof. Since $J$ is underallocated, Lemma 2 says that there can be at most $m|W| /(6 \gamma)$ jobs with window $W$ or nested inside $W$. By definition, no window smaller than $6 \gamma$ contains any jobs. The worry is that the ceilings add too many jobs to one machine. But there are at most $2|W| /(6 \gamma)$ windows nested inside $W$, and the ceilings add at most 1 job to each of these windows. So the total number of jobs in $J^{\prime}$ with windows inside $W$ is at most $|W| /(6 \gamma)+2|W| /(6 \gamma)=|W| /(2 \gamma)$. Even if all jobs are restricted to run at multiples of $\gamma$, a simple inductive argument shows that this many size- $\gamma$ jobs can be feasibly scheduled.

\section{REALLOCATING ALIGNED JOBS ON ONE MACHINE}

We now give a single machine, reallocating scheduler for unitsized jobs. We assume a bound $n$ on the number of jobs concurrently scheduled in the system, and relax this assumption at the end of the section.

\section{Naïve Pecking-Order Scheduling is Logarith- mic}

We first give the naïve solution, which requires a logarithmic number of reallocations per job insert/delete. This solution uses what we call pecking-order scheduling, which means that a job $k$ schedules itself without regard for jobs with longer span and with complete deference to jobs with shorter span. A job $k$ with window $W$ may get displaced by a job $j$ with a shorter window (nested inside $W$ ), and $k$ may subsequently displace a job $\ell$ with longer window. ${ }^{2}$

LEMMA 4. Let $n$ denote the maximum number of jobs in any schedule and let $\Delta$ denote the longest window span. There exists a greedy reallocating scheduler such that for every feasible sequence of recursively aligned scheduling requests, the reallocation cost of each insert/delete is $O(\min \{\log n, \log \Delta\})$.

PROOF. To insert a job $j$ with span $2^{i}$, find any empty slot in $j$ 's window, and place $j$ there. Otherwise, select any job $k$ currently scheduled in $j$ 's window that has span $\geq 2^{i+1}$. If no such $k$ exists, the instance is not feasible (as every job currently scheduled in $j$ 's window must be scheduled in $j$ 's window). If such a $k$ exists, replace $k$ with $j$ and recursively insert $k$. This strategy causes cascading reallocations through increasing window spans, reallocating at most one job with each span. Since there are at most $\log \Delta$ distinct window spans in the aligned case, and moreover all jobs can fit within a window of span $n$, the number of cascading reallocations is $O(\min \{\log n, \log \Delta\})$.

\footnotetext{
${ }^{2}$ At first glance, Lemma 4 seems to contradict the underallocation requirement given in Lemma 12. That lower bound, however, applies to the general case, whereas this lemma applies to the aligned case.
}

\section{Pecking-Order Reallocation via Reservations Costs $O\left(\min \left\{\log ^{*} n, \log ^{*} \Delta\right\}\right)$}

We now give a more efficient reallocating scheduler, which matches Theorem 1 when the scheduling requests are recursively aligned. The algorithm is summarized for job insertions in Figure 4.

The intuition behind reservation scheduling manifests itself in the process of securing a reservation at a popular restaurant. If higher-priority diners already have reservations, then our reservation is waitlisted. Even if our reservation is "confirmed," a celebrity (or the President, for DC residents) may drop in at the last moment and steal our slot. If the restaurant is empty, or full of low-priority people like graduate students, then our reservation is fulfilled. The trick to booking a reservation at a competitive restaurant is to make several reservations in parallel. If multiple restaurants grant the reservation, we can select one to eat at. If a late arrival steals our slot, no problem, we have another reservation waiting.

Back to our scheduling problem, by spreading out reservations carefully, jobs will only interfere if they have drastically different spans. Our algorithm handles jobs with "long" windows and "short" windows separately, and only a "short" job can displace a long job. The scheduler itself is recursive, so "very short" jobs can displace "short" jobs which can displace "long" jobs, but the number of levels of recursion here will be $\log ^{*} \Delta$, as opposed to $\log \Delta$ in the naive solution.

There are two components to the scheduler. The first component uses reservations to guarantee that jobs cannot displace (many) other jobs having "similar" span, so the reallocation cost if all jobs have similar spans is $O(1)$. These (over-)reservations, however, consume timeslots and amplify the underallocation requirements. Applying the scheduler recursively at this point is trivial to achieve a good reallocation cost, but the required underallocation would become nonconstant. The second component of the scheduler is to combine levels of granularity so that their effects on underallocation do not compound.

The remainder of the section is organized as follows. We first discuss an interval decomposition to separate jobs into different "levels" according to their spans. Then we present the scheduler with regards to a single job level. Finally we discuss how to incorporate multiple levels simultaneously.

\section{Interval Decomposition}

Our scheduler operates nearly independently at multiple levels of granularity. More precisely, we view these levels from bottom up by defining the threshold

$$
L_{\ell+1}=\left\{\begin{array}{ll}
2^{5} & \text { if } \ell=0 \\
2^{L_{\ell} / 4} & \text { if } \ell>0
\end{array} .\right.
$$

It is not hard to see that $L$ is always a power of 2, growing as a tower function of $\sqrt[4]{2}$. It is often convenient to use the equivalent relationship $L_{\ell}=4 \lg \left(L_{\ell+1}\right)$ - each threshold is roughly the $\lg$ of the next.

Our scheduler operates recursively according to these thresholds. The level- $\ell$ scheduler handles jobs and windows $W$ with $\operatorname{span} L_{\ell}<$ $|W| \leq L_{\ell+1}$. We call a job (or window) a level- $\ell$ job (window) if its span falls in this range.

We partition level- $\ell$ windows into nonoverlapping, aligned subwindows called level- $\ell$ intervals, consisting of $L_{\ell}=4 \lg L_{\ell+1}$ timeslots. The following observation is useful in our analysis:

(\# of distinct level- $\ell$-window spans $) \leq \lg \left(L_{\ell+1}\right)=L_{\ell} / 4$

The reallocation scheduler operates recursively within each interval to handle lower-level jobs. Because this is pecking-order 
scheduling, the recursive scheduler makes decisions without paying attention to the location of the higher-level jobs, guaranteeing only that each lower-level job is assigned a unique slot within its appropriate window. In doing so, it may displace a long job and invoke the higher-level scheduler.

\section{Schedule Level- $\ell$ Jobs via Reservations}

Consider a level- $\ell$ window $W$ with span $2^{k} L_{\ell}$, for some integer $k \geq 1$ (i.e., $W$ contains $2^{k}$ level- $\ell$ intervals). Let $x$ denote the number of jobs having exactly window $W$.

The window $W$ maintains a set of reservations for these $x$ jobs, where each reservation is a request for a slot in a given level- $\ell$ interval. A reservation made by $W$ can be fulfilled; this means that one slot from the requested interval is assigned to $\boldsymbol{W}$, and the only level- $\ell$ jobs that may occupy that slot are any of the $x$ jobs with window exactly $W$. Alternatively, a reservation can be waitlisted; this means that all the slots in the requested interval are already assigned to smaller windows than $W$. Which reservations are fulfilled and which are waitlisted may change over time as jobs get allocated and removed.

We now explain how these reservations are made. Initially, a level- $\ell$ window $W$ makes one reservation for each enclosed level- $\ell$ interval. It makes two additional reservations for each job having window $W$. These reservations are spread out round-robin among the intervals within $W$ (and independently of any jobs with any different windows). We maintain the following invariant:

INVARIANT 5. If there are $x$ jobs having level- $\ell$ window $W$ with $|W|=2^{k} L_{\ell}$, then $W$ has exactly $2 x+2^{k}$ reservations in level- $\ell$ intervals.

- These reservations are assigned in round-robin order to the intervals in $W$.

- Each of the enclosed intervals contains either $\left\lfloor 2 x / 2^{k}\right\rfloor+1$ or $\left\lfloor 2 x / 2^{k}\right\rfloor+2$ of $W$ 's reservations, where the leftmost intervals have the most reservations and the rightmost intervals have the least reservations.

To maintain Invariant 5, when a new job with window $W$ is allocated, $W$ makes two new reservations, and these are sent to the leftmost intervals that have the least number $\left(\left\lfloor 2 x / 2^{k}\right\rfloor+1\right)$ of $W$ 's reservations. When a job having window $W$ is deleted, $W$ removes one reservation each from the two rightmost intervals that have the most reservations.

We now describe the reservation process from the perspective of the interval, which handles reservation requests from the $<L_{\ell} / 4$ level- $\ell$ windows that contain the interval (see Equation 1). The interval decides whether to fulfill or waitlist a reservation, prioritizing reservations made by shorter windows. Each interval $I$ has an allowance allowance $(I)$, specifying which slots it may use to fulfill reservations. In the absence of lower-level jobs, the $\mid$ allowance $(I) \mid=L_{\ell}$, since the interval has span $L_{\ell}$. (When lowerlevel jobs are introduced, however, the allowance decreasesthe allowance contains all those slots that are not occupied by lower-level jobs.) Thus, the interval sorts the window reservations with respect to span from shortest to longest, and fulfills the $\mid$ allowance $(I) \mid \leq L_{\ell}$ reservations that originate from the shortest windows. A fulfilled reservation is assigned to a specific slot in the interval, while a waitlisted reservation has no slot. The interval maintains a list of these waitlisted reservations.

The set of fulfilled reservations changes dynamically as insertions/deletions occur. When a new reservation is made by window $W$, a longer window $W^{\prime}$ may lose a reserved slot as one of its fulfilled reservations is moved to the waitlist; if there is a job (of the same level) in that slot, it must be moved. When a job with window $W$ is deleted, $W$ has two fewer reservations, and so may lose two fulfilled slots. If there is a job in either of these slots, then that job must be moved. (In this case, a longer window $W^{\prime}$ may gain a fulfilled slot, but this does not require any job movement.) The following invariant is needed to establish the algorithm's correctness.

INVARIANT 6. When a job having window $W$ is newly allocated, $W$ makes two new reservations. Then the job is assigned to any empty slot for which $W$ has a fulfilled reservation. There will always be at least one such slot (proved by Lemma 8).

Interestingly, as a consequence of pecking-order scheduling combined with round-robin reservations:

OBSERVATION 7. Which reservations in which intervals are fulfilled and which are waitlisted is history independent. The actual placement of the jobs is not history independent.

\section{Scheduling Across All Levels}

Consider inserting a level- $\ell$ job $j$. Suppose $j$ 's window is contained in a higher-level interval $I^{\prime}$. We schedule $j$ at its own level according to the pecking-order scheduler, without regard to higher-level schedulers. Recall that the first step of the insertion is placing two new reservations. Whenever the reservations cause another level- $\ell$ job $j^{\prime}$ to move from slot $s$ to slot $s^{\prime}$, the allowance of all higherlevel intervals must be updated to reflect the change in slot usage. However, since both $s \in I^{\prime}$ and $s^{\prime} \in I^{\prime}$, and $j^{\prime}$ vacates the original slot $s$, there is no net change to $\mid$ allowance $\left(I^{\prime}\right) \mid$. It is thus sufficient to swap $s$ and $s^{\prime}$ for all higher-level intervals $I^{\prime}$, which may result in a total of one higher-level job movement.

After updating the reservations, the new job $j$ is placed in one of its assigned slots $s$. This slot may either be empty, or it may contain a higher-level job $h$-the scheduler chooses $s$ without regard to these possibilities. In either case, the slot $s$ will be used by $j$, so it must be removed from allowance $\left(I^{\prime}\right)$ for any ancester interval $I^{\prime}-$ meaning the higher-level scheduler cannot use this slot. If the slot $s$ was empty, then the job $j$ is assigned to that slot and the insertion terminates. If the slot $s$ was previously occupied by a higher-level job $h$, then $h$ is displaced and a new slot must be found. Unlike in the case of reservations, $\mid$ allowance $\left(I^{\prime}\right) \mid$ decreases here and we do not immediately have a candidate slot into which to place $h$. Instead, we reinsert $h$ recursively using the scheduler at its level. This displacement and reinsertion may cascade to higher levels.

Observe that the higher-level scheduler is unaware of the reservation system employed by the lower-level scheduler. It only knows which slots are in its allowance. These slots are exactly those that are not occupied by short-window jobs. The interval does not observe the reservations occurring within nested intervals-only actual job placement matters. When a lower-level job is deleted, the allowance of the containing interval increases to include the slot that is no longer occupied.

\section{Reservation Analysis}

We now use the following lemma to establish Invariant 6, which claims that there are always enough fulfilled reservations. Since the reservations fulfilled by each interval are history independent (see Observation 7), this proof applies at all points during the execution of the algorithm.

LEMMA 8. Suppose that a sequence of aligned scheduling requests 8-underallocated. If there are $x$ jobs each having the same window $W$, then $W$ has at least $x+1$ fulfilled reservations. 
- Initially, each level- $\ell$ window $W$ has one reservation in each level- $\ell$ interval contained in $W$.

- Initially, each interval $I$ has allowance $(I)=I$.

- To insert a new level- $\ell$ job $j$ with window $W$ :

1. Identify the two underloaded intervals $I_{1}$ and $I_{2}$ according to Invariant 5

2. Call $\operatorname{Reserve}\left(I_{1}, W\right)$ and $\operatorname{Reserve}\left(I_{2}, W\right)$

3. Call PLACE $(j)$

$\operatorname{ReserVE}(I, W)$

$/ /$ make a reservation in $I$ for level- $\ell$ window $W$

1 if there is a slot $s \in$ allowance $(I)$ that has not been assigned

then fulfill the reservation, assigning slot $s$ to window $W$ and return

3 let $W^{\prime}$ be the longest window with a fulfilled reservation in $I$, and let $s$ be one of its slot

if $\left|W^{\prime}\right| \leq|W|$

then waitlist the reservation for $W$

else waitlist the reservation for $W^{\prime}$ and take slot $s$ from $W^{\prime}$

if there is a level- $\ell$ job $j^{\prime}$ in slot $s$

then $\operatorname{Move}\left(j^{\prime}\right)$

fulfill the reservation, assigning slot $s$ to $W$

// Note that though the reservation is fulfilled, the slot may be occupied by a higher-level job

$\operatorname{Move}\left(j^{\prime}\right)$

// level- $\ell$ job $j^{\prime}$ lost the reservation to a slot it occupies

10 let $W^{\prime}$ be the window of $j^{\prime}$, and let $s$ be the slot it occupies

11 let $s^{\prime}$ be a fulfilled slot, assigned to $W^{\prime}$, not containing any level- $\ell$ job

12 for all ancestor intervals $I^{\prime}$ containing $W^{\prime}$

13 do swap $s$ and $s^{\prime}$ with regards to reservations and allowances for $I^{\prime}$

// if a higher-level job $h$ occupies $s^{\prime}$ then schedule $h$ in $s$ instead of $s^{\prime}$

14 schedule $j^{\prime}$ in slot $s^{\prime}$

$\operatorname{Place}(j)$

15 let $s$ be a fulfilled slot, assigned to $W$, not containing any level- $\ell$ job

16 schedule $j$ in $s$, potentially displacing a higher-level job $h$

17 remove $s$ from the allowance of all higher-level intervals

18 for each ancestor interval whose allowance decreases

do adjust the reservations to reflect a smaller allowance, possibly waitlisting one reservation

if a newly waitlisted reservation is for a slot containing a job $j^{\prime}$

then $\operatorname{Move}\left(j^{\prime}\right)$

if there is a displaced job $h$

then $\operatorname{Place}(h)$

// let $W$ be $j$ 's window and let $\ell$ be $j$ 's level

// exists by Lemma 8

// $s$ is only in allowances up to $h$ 's level

19

20

22

23

// exists by Lemma 8

// both slots are inside $I^{\prime}$

Figure 1: Pecking-order scheduling with reservations.

Proof. Let $|W|=2^{k} L_{\ell}$ for level- $\ell$ window $W$. Let $y$ be the number of level- $\ell$ jobs with windows nested inside $W$. Each of those windows makes 2 reservations for each job, plus an extra reservation to each of the $2^{k}$ intervals. So the total number of reservations in $W$ is at most $2(x+y)+2^{k} \lg W$. In addition, let $z$ be the number of lower-level jobs nested inside $W$. Since we are 8underallocated, we have $2(x+y)+z \leq 2(x+y+z) \leq|W| / 4$ by Lemma 2 . By Equation 1, we have $\lg W \leq L_{\ell} / 4$, and hence $2^{k} \lg W \leq\left(2^{k} L_{\ell}\right) / 4=|W| / 4$. Summing these up, we have that at most $|W| / 2$ slots consumed by lower-level jobs and these reservations.

In order for a particular interval to waitlist even one of $W$ 's reservation requests, it would need to have strictly more than $L_{\ell}$ of these reservations or lower-level jobs assigned to it. But there are only $|W| / 2$ slots consumed in total, so strictly less than $1 / 2$ the intervals can waitlist even one of $W$ 's reservations. Since window $W$ reserves at least $\left\lfloor 2 x / 2^{k}\right\rfloor+1$ slots in every one of the $2^{k}$ intervals by Invariant 5 , it must therefore be granted strictly more than $\left(\left\lfloor 2 x / 2^{k}\right\rfloor+1\right)(1 / 2)\left(2^{k}\right) \geq x$ fulfilled reservations.
Since each window $W$ containing $x$ jobs has at least $x+1$ fulfilled reservations at intervals within $W$, there is always an appropriate slot to schedule a new belonging to this window. This ensures that there each operation leads to only $O(1)$ reallocations at each level.

\section{Trimming Windows to $\boldsymbol{n}$ and Deamortization}

Ideally, the reallocation cost for a request $r$ should be a function of the number of active jobs $n_{r}$ in the system when request $r$ is made. To achieve this performance guarantee, we maintain a value $n^{*}$ that is roughly the number of jobs in the current schedule. When the number of active jobs exceeds $n^{*}$, we double $n^{*}$; when the number of active jobs shrinks below $n^{*} / 4$, we halve $n^{*}$.

For every job that has a window larger than $2 \gamma n^{*}$, we trim its window-reducing it arbitrarily to size $2 \gamma n^{*}$. The adjusted instance remains $\gamma$-underallocated, since there are at most $n^{*}$ other jobs scheduled in the trimmed window of size $2 \gamma n^{*}$.

To achieve good amortized performance, it is enough to rebuild the schedule from scratch each time we change the value of $n^{*}$. This rebuilding incurs an amortized $O(1)$ reallocation cost. 
This amortized solution can be deamortized, as long as the scheduling instance is sufficiently underallocated that the following property holds: if each job is duplicated (i.e., inserted twice on inserts, deleted twice on delete), the resulting instance is $\gamma$ underallocated, for appropriate constant $\gamma$. This property holds as long as the initial (unduplicated) scheduling instance is $2 \gamma$ underallocated.

The idea is to rebuild the schedule gradually, performing a little update every time a new reallocation request is serviced. This approach is reminiscent to how one deamortizes the rebuilding of a hash table that is too full or too empty. We use the even (or odd) time slots for the old schedule and the odd (or even) time slots for the new schedule. Instead of rebuilding the schedule all at once, every time one job is added or deleted, two jobs are moved from the old schedule to the new schedule.

\section{Wrapping Up}

We conclude with the following lemma, which puts together the various results in this section:

LEMMA 9. There exists a constant $\gamma$ and a single-machine reallocating scheduler such that for any 1-machine $\gamma$-underallocated sequence of aligned scheduling requests, we achieve the following performance. Let $n_{i}$ denote the number of jobs in the schedule and $\Delta_{i}$ the largest window size when the ith reallocation takes place. Then the ith reallocation has cost $O\left(\min \left\{\log ^{*} n_{i}, \log ^{*} \Delta_{i}\right\}\right)$.

PROOF. We consider the performance of the pecking-order scheduler with reservations, where we maintain an estimate $n^{*}$ via deamortized shrinking and doubling and trim all windows to $\gamma n^{*}$.

Lemma 8 shows that there is always a slot available to put a job (Invariant 6), and hence we observe that there are at most $O(1)$ reallocations at each level of the scheduler. Specifically, on insertion, the two reservations may result in two calls to Move for jobs at the same level as the one being inserted. Each MOVE results in one reallocation of the job being moved, plus at most one reallocation at a higher level. Then the call to PLACE may cascade across all levels, but it in aggregate it only includes one MOVE per level, each causing at most two reallocations.

If $\Delta_{i}$ is the largest job size when operation $i$ occurs, there are no more than $O\left(\log ^{*} \Delta_{i}\right)$ levels. Since $n_{i}^{*} \leq 4 n$, and all windows are trimmed to length $\gamma n^{*}$, we also know that there are no more than $O\left(\log ^{*}\left(4 \gamma n_{i}\right)\right)$ levels. From this the result follows.

\section{REALLOCATING UNALIGNED JOBS ON MULTIPLE MACHINE}

In this section, we generalize to jobs that are not aligned, removing the alignment assumptions that we made in Sections 3 and 4. We show that if $S$ is a $\gamma$-underallocated sequence of scheduling requests, then we can safely trim each of the windows associated with each of the jobs, creating an aligned instance. Since the initial sequence of scheduling requests is underallocated, the resulting aligned sequence is also underallocated, losing only a constant factor.

We first define some terminology. If $W$ is an arbitrary window, we say that $\operatorname{ALIGNED}(W)$ is a largest aligned window that is contained in $W$. (If there is more than one largest window, choose arbitrarily.) Notice that $|\operatorname{ALIGNed}(W)| \geq|W| / 4$. If $J$ is a set of jobs, then $\operatorname{ALIGNED}(J)$ is the set of jobs in which the window $W$ associated with each job is replaced with $\operatorname{ALIGNED}(W)$.

LEMMA 10. Consider any m-machine $4 \gamma$-underallocated set of jobs $J$, where $\gamma$ is an integer. Then $\operatorname{ALIGNED}(J)$ is $m$-machine $\gamma$-underallocated.
Proof. Assume for the sake of contradiction that $\operatorname{ALIGNED}(J)$ is not $\gamma$-underallocated. This implies that there must exist a window $W$ that has $>m|W| / \gamma$ jobs with trimmed windows contained in $W$ (as otherwise we could schedule the size $\gamma$ jobs via a simple inductive argument). Let $J^{\prime} \subseteq J$ be the jobs whose trimmed windows are contained in $W$.

Since $J$ is $4 \gamma$-underallocated, we now examine an (unaligned) scheduling of the jobs in $J^{\prime}$ that satisfies the $4 \gamma$-underallocation requirement. We observe that all the jobs in $J^{\prime}$ are scheduled in a region of size at most $4|W|$. However, since the schedule is $4 \gamma$-underallocated, there can be at most $4 m|W| /(4 \gamma)$ jobs in this region of size $4|W|$. That is $\left|J^{\prime}\right| \leq m|W| / \gamma$, which is a contradiction.

From this we can conclude with the proof of Theorem 1:

PROOF OF THEOREM 1. Jobs are scheduled as follows: first, a new job has its window aligned; second, it is delegated to a machine in round-robin fashion; finally, it is scheduled via single-machine pecking-order scheduling with reservations. When a job is deleted, it is removed by the appropriate single-machine scheduler, and then there is at most one migration to maintain the balance of jobs across machines. This is the only time that jobs migrate.

Lemma 10 shows that the set of aligned jobs is $m$-machine $\gamma / 4$-underallocated, and Lemma 3 shows that the jobs asigned to each machine are 1-machine $\gamma / 24$-underallocated. Finally, Lemma 9 shows that each single-machine scheduler operation has cost $O\left(\min \left\{\log ^{*} n_{i}, \log ^{*} \Delta_{i}\right\}\right)$ —and each job addition or deletion invokes $O(1)$ single-machine scheduler operations.

\section{WHAT HAPPENS WITHOUT UNDER- ALLOCATION?}

This section explains what happens without underallocation and why migrations are necessary at all.

If migration cost is to be bounded only by reallocation cost and since jobs have unit size, it is trivial to transform a parallel instance to a single-machine instance my making a single machine go $m$ times faster. Since migration cost across machines could be more expensive than rescheduling a single machine, we are interested in providing a tighter bound on the migration cost. The question then is: are migrations necessary? The following lemma shows that they are. In fact, the per-request migration cost must be $\Omega(1)$ in the worst-case for any deterministic algorithm.

LEMMA 11. There exists a sufficiently large sequence of $s$ job insertions/deletions on $m>1$ machines, such that any deterministic scheduling algorithm has a total migration cost of $\Omega(s)$.

Proof. Without loss of generality, assume $6 \mathrm{~m}$ divides $s$. Divide the $s$ requests into $s /(6 \mathrm{~m})$ consecutive subsequences of $6 \mathrm{~m}$ requests each. Each subsequence is as follows:

1. Insert $2 m$ span-2 jobs with window $[0,2]$.

2. Delete the $m$ jobs scheduled on the first $m / 2$ machines.

3. Insert $m$ span-1 jobs with windows $[0,1]$.

4. Delete all $2 m$ remaining jobs.

After step 1, the only feasible schedule is to put two jobs on each machine. After step 2, half the machines have two jobs, and the other half of the machines have no jobs. The only feasible schedule after step 3 is to have on each machine a span-1 job starting at time 0 , and a span- 2 job starting at time 1 . This means that half of the span- 2 jobs must migrate across machines, causing $\mathrm{m} / 2 \mathrm{mi}$ grations. There are thus $m / 2$ migrations for every $6 m$ requests, or a total of $s / 12$ migrations. 
It is also easy to see that for some sequences of scheduling requests, if they are not underallocated, it is impossible to achieve low reallocation costs, even if there exists a feasible schedule.

LEMMA 12. There exists a sequence of s job inserts/deletions, such that any scheduling algorithm has a rescheduling cost of $\Omega\left(s^{2}\right)$.

Proof. Consider for example $\eta=s / 2$ jobs numbered $0,1, \ldots, \eta-1$, where job $j$ has window $[j, j+2]$. With the insertion of one additional job having window $[0,1]$, forcing the job to be scheduled at time 0 , all $\eta$ other jobs are forced to schedule during their later slot. If that job is deleted and another unit-span job with window $[\eta, \eta+1]$ is inserted, then all jobs are forced to schedule during their earlier slot. By toggling between these two options, all jobs are forced to move, resulting in cost $\Omega(\eta)$ to handle each request. Repeating $\eta$ times gives a total cost of $\Omega\left(\eta^{2}\right)=\Omega\left(s^{2}\right)$.

\section{CONCLUSIONS AND OPEN QUES- TIONS}

The results in this paper suggest several followup questions. First, is it possible to generalize this paper's reallocation scheduler for the case where jobs are not unit-sized? Observe that we are limited by the computational difficulty of scheduling with arrival times and deadlines when jobs are not unit size; see [6] for recent results with resource augmentation. We are also limited by the following observation:

OBSERVATION 13. Suppose there exist jobs of size 1 and jobs of size $k$, for any $k>1$. For any reallocation scheduler, there is a sequence of $\Theta(n)$ scheduling requests that has aggregate reallocation cost $\Omega(k n)$, for $k \leq n$, even if the requests are $\gamma$ underallocated for any constant $\gamma$.

Proof. Consider a schedule of length $m=2 \gamma k$. Assume there are $k$ unit-sized jobs that are each scheduled with a window beginning at 0 and ending at $m$. In addition, consider a single large job $p$ that has size $k$ and a window of span exactly $k$.

Initially, all $k$ unit-size jobs are scheduled and they remain in the system throughout. The large job $p$ is initially scheduled at time slot 0 . It is then deleted from time slot 0 and re-inserted at time slot $k$, and then again at time slot $2 k, 3 k, \ldots, m-k$. The same sequence of $2 \gamma$ insertions and deletions is then repeated $n$ times.

During a single sequence of $2 \gamma$ insertions and deletions, each of the $k$ unit-sized jobs has to be rescheduled at least once, resulting in $\Omega(k n)$ reallocation cost.

Does there exist a reallocation scheduler that handles jobs whose sizes are integers less than or equal to $k$ and matching the bounds in Observation 13? There could be applications where jobs are not unit size, but where $k$ is relatively small.

What happens if other types of reallocations are allowed, such as if new machines can be added or dropped from the schedule, or if machine speeds can change?

In this paper, $\gamma$ is very large, and the paper does not attempt to optimize this constant, preferring clarity of exposition. How much can this constant be improved? Is there a reallocation scheduler where $\gamma=1+\varepsilon$ ?

Finally, what other scheduling and optimization problems lend themselves to study in the context of reallocation?

\section{REFERENCES}

[1] C. Archetti, L. Bertazzi, and M. G. Speranza. Reoptimizing the Traveling Salesman Problem. Networks, 42(3):154-159, 2003.
[2] C. Archetti, L. Bertazzi, and M. G. Speranza. Reoptimizing the 0-1 knapsack problem. Discrete Appl. Math., 158(17):1879-1887, Oct. 2010.

[3] G. Ausiello, V. Bonifaci, and B. Escoffier. Complexity and approximation in reoptimization. In Proccedings of CiE: Logic and Computation and Logic in the Real World, 2007.

[4] G. Ausiello, B. Escoffier, J. Monnot, and V. T. Paschos. Reoptimization of minimum and maximum traveling salesman's tours. J. Discrete Algorithms, 7(4):453-463, 2009.

[5] B. Awerbuch, Y. Azar, S. Leonardi, and O. Regev. Minimizing the flow time without migration. SIAM J. Comput., 31(5):1370-1382, 2002.

[6] N. Bansal, H.-L. Chan, R. Khandekar, K. Pruhs, C. Stein, and B. Schieber. Non-preemptive min-sum scheduling with resource augmentation. In Proc. 48th Annual IEEE Symposium on Foundations of Computer Science (FOCS), pages 614-624, 2007.

[7] L. Becchetti, S. Leonardi, and S. Muthukrishnan. Average stretch without migration. J. Comput. Syst. Sci., 68(1):80-95, 2004.

[8] M. A. Bender, J. T. Fineman, and S. Gilbert. A new approach to incremental topological ordering. In Proc. 20th ACM-SIAM Symposium on Discrete Algorithms (SODA), pages 1108-1115, January 2009.

[9] M. A. Bender and H. Hu. An adaptive packed-memory array. Transactions on Database Systems, 32(4), 2007.

[10] H.-J. Böckenhauer, L. Forlizzi, J. Hromkovic, J. Kneis, J. Kupke, G. Proietti, and P. Widmayer. Reusing optimal TSP solutions for locally modified input instances. In Proc. Fourth IFIP International Conference on Theoretical Computer Science (TCS), pages 251-270, 2006.

[11] A. Caprara, L. Galli, L. Kroon, G. Maróti, and P. Toth. Robust train routing and online re-scheduling. In T. Erlebach and M. Lübbecke, editors, Proc. 10th Workshop on Algorithmic Approaches for Transportation Modelling, Optimization, and Systems, volume 14 of OpenAccess Series in Informatics (OASIcs), pages 24-33, Dagstuhl, Germany, 2010. Schloss Dagstuhl-Leibniz-Zentrum fuer Informatik.

[12] V. Chiraphadhanakul and C. Barnhart. Robust flight schedules through slack re-allocation. Submitted, 2011.

[13] S. Davis, J. Edmonds, and R. Impagliazzo. Online algorithms to minimize resource reallocations and network communication. In APPROX-RANDOM, pages 104-115, 2006.

[14] S. P. Fekete, T. Kamphans, N. Schweer, C. Tessars, J. C. van der Veen, J. Angermeier, D. Koch, and J. Teich. Dynamic defragmentation of reconfigurable devices. ACM Trans. Reconfigurable Technol. Syst., 5(2):8:1-8:20, June 2012.

[15] B. Haeupler, T. Kavitha, R. Mathew, S. Sen, and R. E. Tarjan. Faster algorithms for incremental topological ordering. In Proc. 35th International Colloquium on Automata, Languages, and Programming (ICALP), pages 421-433, July 2008.

[16] N. G. Hall and C. N. Potts. Rescheduling for new orders. Operations Research, 52(3), 2004.

[17] A. Itai, A. G. Konheim, and M. Rodeh. A sparse table implementation of priority queues. In Proc. 8th Internationl Colloquium on Automata, Languages, and Programming (ICALP), volume 115 of Lecture Notes in Computer Science, pages 417-431, 1981. 
[18] J. Jackson. Scheduling a production line to minimize maximum tardiness. Technical report, Management Science Research Project Research Report 43, University of California, Los Angeles, 1955.

[19] H. Jiang and C. Barnhart. Dynamic airline scheduling. Transportation Science, 43(3):336-354, 2009.

[20] B. Kalyanasundaram and K. Pruhs. Speed is as powerful as clairvoyance. Journal of the ACM, 47:214-221, 1995.

[21] I. Katriel and H. L. Bodlaender. Online topological ordering. In Proc. 16th ACM-SIAM Symposium on Discrete Algorithms (SODA), pages 443-450, Vancouver, British Columbia, Canada, January 2005.

[22] P. Kouvelis and G. Yu. Robust Discrete Optimization and Its Applications. Kluwer, 1997.

[23] S. Lan, J.-P. Clarke, and C. Barnhart. Planning for robust airline operations: Optimizing aircraft routings and flight departure times to minimize passenger disruptions. Transportation Science, 40(1):15-28, 2006.

[24] J. M. Mulvey, R. J. Vanderbei, and S. A. Zenios. Robust optimization of large-scale systems. Operations Research, 43(2), 1995.

[25] C. A. Phillips, C. Stein, E. Torng, and J. Wein. Optimal time-critical scheduling via resource augmentation. Algorithmica, 32(2):163-200, 2002.

[26] P. Sanders, N. Sivadasan, and M. Skutella. Online scheduling with bounded migration. Math. Oper. Res., 34(2):481-498, 2009.

[27] H. Shachnai, G. Tamir, and T. Tamir. A theory and algorithms for combinatorial reoptimization. In Proc. 10th Latin American Symposium Theoretical Informatics (LATIN), pages 618-630, 2012.

[28] C. A. Tovey. Rescheduling to minimize makespan on a changing number of identical processors. Naval Research Logistics, 33:717-724, 1986.

[29] A. T. Unal, R. Uzsoy, and A. S. Kiran. Rescheduling on a single machine with part-type dependent setup times and deadlines. Annals of Operations Research, 70, 1997.

[30] J. Westbrook. Load balancing for response time. Journal of Algorithms, 35(1):1 - 16, 2000.

[31] D. Willard. Maintaining dense sequential files in a dynamic environment (extended abstract). In Proc. 14th Annual Symposium on Theory of Computing (STOC), pages 114-121, 1982.

[32] D. E. Willard. Good worst-case algorithms for inserting and deleting records in dense sequential files. In Proc. International Conference on Management of Data (SIGMOD), pages 251-260, 1986.

[33] D. E. Willard. A density control algorithm for doing insertions and deletions in a sequentially ordered file in good worst-case time. Information and Computation, 97(2):150-204, 1992. 